\section{ADDITIONAL INFORMATION}

Publisher's note Springer Nature remains neutral with regard to jurisdictional claims in published maps and institutional affiliations.

\section{REFERENCES}

1. Rush AJ, et al. Acute and longer-term outcomes in depressed outpatients requiring one or several treatment steps: a STAR*D report. Am J Psychiatry. 2006;163:1905-17.

2. Krishnan V, et al. Molecular adaptations underlying susceptibility and resistance to social defeat in brain reward regions. Cell. 2007;131:391-404.

3. Hultman R, et al. Dysregulation of Prefrontal Cortex-Mediated Slow-Evolving Limbic Dynamics Drives Stress-Induced Emotional Pathology. Neuron. 2016;91:439-52.

4. Hultman R. et al. Brain-wide Electrical Spatiotemporal Dynamics Encode Depression Vulnerability. Cell. 2018;173:166-180.e14.

5. Nakayama R, Ikegaya Y, Sasaki T. Cortical-wide functional correlations are associated with stress-induced cardiac dysfunctions in individual rats. Sci Rep. 2019;9:10581. https://doi.org/10.1038/s41598-019-47171-y
6. Drysdale AT, et al. Resting-state connectivity biomarkers define neurophysiological subtypes of depression. Nat Med. 2017;23:28-38.

(i) Open Access This article is licensed under a Creative Commons Attribution 4.0 International License, which permits use, sharing, adaptation, distribution and reproduction in any medium or format, as long as you give appropriate credit to the original author(s) and the source, provide a link to the Creative Commons license, and indicate if changes were made. The images or other third party material in this article are included in the article's Creative Commons license, unless indicated otherwise in a credit line to the material. If material is not included in the article's Creative Commons license and your intended use is not permitted by statutory regulation or exceeds the permitted use, you will need to obtain permission directly from the copyright holder. To view a copy of this license, visit http://creativecommons. org/licenses/by/4.0/.

(c) The Author(s) 2019

\title{
MDMA-assisted psychotherapy for posttraumatic stress disorder: A promising novel approach to treatment
}

\author{
Eric Vermetten (1D) ${ }^{1,2}$ and Rachel Yehuda ${ }^{3,4}$ \\ Neuropsychopharmacology (2020) 45:231-232; https://doi.org/10.1038/s41386-019-0482-9
}

Posttraumatic stress disorder (PTSD) treatment guidelines have unequivocally designated psychotherapy as a first-line treatment, despite well-documented neurobiological alterations in this disorder [1]. Even with psychotherapy, PTSD often remains chronic and severe. There is urgency to discover novel compounds and new treatment strategies for PTSD.

One approach is the use of medication to leverage the effects of psychotherapy. A promising example is the use of 3,4-methylenedioxymethamphetamine (MDMA). MDMA was synthesized in 1912 by Merck and discovered in the early 1970 s to enhance effects of psychotherapy. The subsequent classification of MDMA as a Schedule 1 controlled substance made its use in therapy illegal and created obstacles to clinical research, including assessment of safety.

Nonetheless, new research has emerged demonstrating the efficacy of MDMA as an enhancer of psychotherapy for PTSD. Recently, a pooled analysis was published from six small randomized, double-blind, controlled clinical trials of MDMA [2]. Patients were enrolled to manualized psychotherapy sessions in two or three $8 \mathrm{~h}$ sessions, spaced a month apart. They were given either active doses of MDMA ranging from 75 to $125 \mathrm{mg}(n=72)$ or placebo/ control doses $0-40 \mathrm{mg}(n=31)$. Non-drug sessions lasting $90 \mathrm{~min}$ preceded the first MDMA exposure and three to four weekly sessions following the drug-facilitated session. Two clinicians facilitated an introspective process in which the patient revisited past experiences while under a mental state produced by MDMA that presumptively minimized fear, arousal, and avoidance of painful material. Under MDMA, patients experienced significantly greater reductions in PTSD symptom scores than under placebo, with a treatment effect of 0.8. After two experimental sessions, double the participants in the active group (54.2\%) did not meet PTSD diagnostic criteria than in the control group (22.6\%). Based on these pooled results Food and Drug Administration (FDA) granted MDMA a breakthrough therapy designation for the treatment of PTSD. Yet, it will be important to continue to assess safety of MDMA, particularly when higher or multiple doses are used [3].

MDMA-assisted psychotherapy provides a novel approach for examining how the use of a medication that dramatically alters a cognitive state can facilitate a deeper psychotherapeutic process [4]. It offers a contrast to the current use of medications and psychotherapy, which are often not well integrated and/or provided under the auspices of a single clinician. The fewer in number, but lengthier sessions in the presence of MDMA also redefine concepts regarding the appropriate approach and length of a therapy session involving engagement with traumatic material [5].

Working with FDA in America and the European Medicines Agency in Europe, the pooled data formed the basis for expansion into multi-site Phase 3 trials of MDMA therapy for PTSD. These are sponsored by a non-profit organization that has raised funds through philanthropy, which offers yet another model of drug and therapeutic development. Study centers in the United States are now underway. The European sites-in the Netherlands, United Kingdom, Germany, Finland, Portugal and the Czech Republic-are in the process of seeking approvals and are projected to start later in 2019, putting MDMA on course to becoming a licensed treatment in 2021 [6].

\footnotetext{
${ }^{1}$ Department Psychiatry, Leiden University Medical Center, Leiden, The Netherlands; ${ }^{2}$ ARQ National Psychotrauma Center, Diemen, The Netherlands; ${ }^{3}$ ames J. Peters Veterans Administration Medical Center, New York, NY, USA and ${ }^{4}$ Department Psychiatry, Mount Sinai University School of Medicine, New York, NY, USA Correspondence: Eric Vermetten (e.vermetten@lumc.nl)
} 


\section{FUNDING AND DISCLOSURE}

No applicable funding. The authors declare no competing interests.

\section{ADDITIONAL INFORMATION}

Publisher's note: Springer Nature remains neutral with regard to jurisdictional claims in published maps and institutional affiliations.

\section{REFERENCES}

1. Yehuda R, Hoge CW, McFarlane AC, Vermetten E, Lanius RA, Nievergelt CM, et al. Post-traumatic stress disorder. Nat Rev Dis Prim. 2015;1:15057.
2. Mithoefer MC, Feduccia AA, Jerome L, Mithoefer A, Wagner M, Walsh Z, et al. MDMA-assisted psychotherapy for treatment of PTSD: study design and rationale for phase 3 trials based on pooled analysis of six phase 2 randomized controlled trials. Psychopharmacology (Berl). 2019;236:2735-45.

3. De la Torre R, Farré $M$, Ortuño J, Mas $M$, Brenneisen R, Roset PN, et al. Non-linear pharmacokinetics of MDMA "exstasy" in humans. Br J Clin Pharm. 2000;49:104-9.

4. Nardou R, Lewis EM, Rothhaas R, Xu R, Yang A, Boyden E, et al. Oxytocin-dependent reopening of a social reward learning critical period with MDMA. Nature. 2019; 569:116.6.

5. Wagner AC, Mithoefer MC, Mithoefer AT, Monson CM. Combining cognitivebehavioral conjoint therapy for PTSD with 3, 4-methylenedioxymethamphetamine (MDMA): a case example. J Psychoact Drugs. 2019;51:166-73.

6. Sessa B, Higbed L, Nutt D. A review of 3, 4-methylenedioxymethamphetamine (MDMA)-assisted psychotherapy. Front Psychiatry. 2019;10:138.

\title{
Spatial transcriptomics: putting genome-wide expression on
} the map

\author{
K. R. Maynard (D) ${ }^{1}$, A. E. Jaffe (D) ${ }^{1,2,3,4,5}$ and K. Martinowich ${ }^{1,4,5}$ \\ Neuropsychopharmacology (2020) 45:232-233; https://doi.org/10.1038/s41386-019-0484-7
}

Extensive efforts are underway to comprehensively characterize changes in the human brain transcriptome in neurodevelopmental and neuropsychiatric disorders. Although bulk RNAsequencing (RNA-seq) in postmortem human brain has identified many molecular associations with psychiatric disease, analysis of homogenate brain tissue can mask the heterogeneity of associations within and across specific cell types. Although computational approaches exist to deconvolute cell-type-specific effects from bulk data, these approaches largely control for cellular heterogeneity across samples [1] and do not pinpoint candidate cell types harboring transcriptional differences [2]. However, rapid progress towards characterizing the cell types that make up the brain has been achieved by major advances in single-cell (sc) and single-nuclei (sn) approaches, which are revealing molecular profiles of distinct cell populations. However, these methods still require tissue dissociation, which removes molecularly defined cell types from their spatial environment. This is problematic, because the ability to assess gene expression as a function of neuroanatomy and cytoarchitectural organization will be critical for interpreting molecular and genetic associations.

Methods such as laser-capture microdissection allow for transcriptome-wide profiling in a defined spatial area, but tissue is removed from the surrounding spatial context, making it difficult to analyze gradients of gene expression. The rapidly accelerating field of spatial transcriptomics utilizes techniques that examine the location of hundreds of gene targets in intact tissue slices. In situ sequencing and fluorescent in situ hybridization-based technologies have achieved high levels of multiplexing in single cells of mouse brain using padlock probes or barcoding strategies in combination with sequential rounds of probing, imaging, and stripping (reviewed in refs. [3, 4]). However, even for methodologies that can accommodate hundreds of transcripts simultaneously, molecular crowding within cells leads to fluorescence overlap posing significant microscopy and computational challenges $[3,5]$.

Although not yet sc in resolution, additional platforms such as Slide-seq [6] and SPATiAL transcriptomics [7] have emerged to provide positional context of genome-wide expression in intact tissue sections. SPATiAL generates transcriptome-wide RNA-seq data through capture of polyadenylated RNA on arrays containing positional molecular barcodes that are introduced during cDNA synthesis, which occurs on the surface of the tissue section. Barcoded CDNA is then cleaved, prepared into libraries, and sequenced on a standard Illumina platform. Each RNA is then mapped back to its spatial location within 1 of 1000 different molecular positions in a $6 \mathrm{~mm}^{2}$ array. Although these approaches have not yet been widely applied to postmortem human brain, SPATiAL has been used to identify perturbations in transcriptional pathways for several normal and pathological human tissues, including the spinal cord [8].

Ultimately, understanding the molecular basis of psychiatric disease will require linking molecularly defined cell types to measures of function, including correlates of morphology, physiology, and connectivity. Analyzing gene expression within the existing tissue architecture in the human brain is the next step. These rapidly evolving spatial transcriptomics techniques open possibilities for combining spatial expression maps with sc/snRNA-seq data using spatial registration approaches to add anatomical dimensions to existing datasets and further refine celltype classifications in the human brain. Importantly, these spatial transcriptomic approaches capture gene expression in the cytoplasm and neurites (Fig. 1), which has been largely missed by snRNA-seq approaches in human brain tissue. This is important, because synaptically localized mRNAs may be especially relevant for understanding genetic risk for psychiatric disorders as

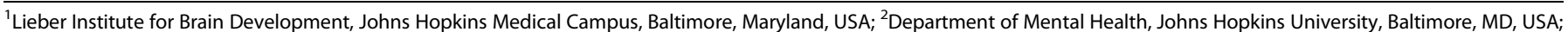

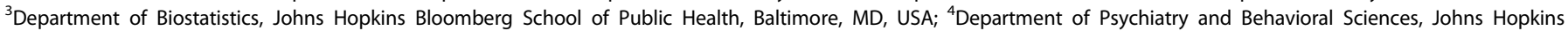
University School of Medicine, Baltimore, MD, USA and ${ }^{5}$ Department of Neuroscience, Johns Hopkins University School of Medicine, Baltimore, MD, USA

Correspondence: K R. Maynard (kristen.maynard@libd.org)
}

Published online: 23 August 2019 